\title{
Design and Implementation of Pharmaceutical Logistics and Supply Chain Management System for Hospital
}

\author{
Kong Hua-li ${ }^{1 \mathrm{a}}$, Feng Duan-hao ${ }^{1 \mathrm{~b}}$, Fan Wei-wei ${ }^{2 \mathrm{c}}$, Li Qian ${ }^{1 \mathrm{~d}}$ \\ ${ }^{1}$ Department of Pharmacy, ${ }^{2}$ Department of Information, The 309th Hospital of PLA, Beijing, 100091, \\ China \\ aemail: xiaojiunengxing@163.com, bemail: fdh62@sohu.com, \\ cemail: 70812770@qq.com, ${ }^{\mathrm{c}}$ email:liqian233244@163.com
}

Keywords: Pharmaceutical Logistics; Supply Chain Management; Internet of Things; Web Service;

\begin{abstract}
Logistics and supply chain management system for hospital pharmacy is an important element of HIS. But the HIS in common use still lack support for drug logistics and supply chain service functionality and documentation management is done manually. There is a information gap between hospital and drug supplier because of traditional HIS. The software described here represents an important step forward to fill this gap. Our system realized real-time tracing and supply chain management of all drugs in hospital by using Internet of Things and Web Services technology. The proposed system is mainly used for information process during drug purchase, request, distribution, acceptance, provision and drug inventory management. It has a significant impact on information management of hospital pharmacy.
\end{abstract}

\section{Introduction}

Real-time tracing and supply chain management of all drugs in hospital is a challenge in healthcare require further developments in information and communication technologies. Indeed, issues such as usability, cooperation, security, interoperability, and consistency of data can directly or indirectly affect work efficiency and patient safety.

The use of manual practices or paper-based documentation in the hospital pharmacy has lead to a series of significant problems, including limitations in record traceability and documentation space, time consuming storage and retrieval of information. All of these factors have unnecessarily increased the workload of pharmacists and is a substantial burden when statistics is needed. Current available approaches often lack in user friendliness, integration with existing systems and devices, workflow and collaborative tasks support. Applying the proposed information system, along with the Internet of Things technology to support the real-time tracing and supply chain management of all drugs in hospital, will alleviate all of these problems and will improve the standard to service to information requesters.

This manuscript is structured as follows. Section II introduces the architecture of system. While section III discusses workflow of system. Section IV describes the Implementation of proposed system. In Section V concludes the paper.

\section{Architecture of System}

The design of logistics and supply chain management system for hospital pharmacy is based on Internet of Things technology(such as wireless network, barcode and mobile terminal etc.). ${ }^{[1]}$ The system realized real-time tracing and supply chain management of all drugs in hospital. The overall design principal is using standardized web service interface to post the hospital pharmacy information which is needed by the logistics and supply chain management system without changing the workflow of existing hospital information system. This system has a significant impact on information management of hospital pharmacy. The system architecture is illustrated in figure 1 . This architecture is made of the following basic components ${ }^{[2]}$ : Pharmacy Application Server; Pharmaceutical Suppliers Web Server; HIS server; Automatic Drug Dispensing System; 
Drug Storage Refrigerator; Pharmacy Mobile Tablet; Nurse Mobile Tablet; Doctor Workstation; Drug Storage Workstation; Pharmacy Workstation; Drug Barcode; Patient Identification Wristband and Net gap etc. Detailed description is as follows:

Pharmacy Application Server:

This server is used to integrate the traditional hospital information systems of medical institution and drug suppliers, provide drug orders to the pharmaceutical supplier web server. Web services running on the server are used to realize the SOA-based heterogeneous data integration in this component. These services provide information exchange between the automatic drug dispensing system, drug storage refrigerator, pharmacy workstation and HIS.

Pharmaceutical Suppliers Web Server:

This server is used for manage the drug suppliers of hospital, the drug purchasing plan is provided by this server, the drug supplier can download the hospital drug orders, then finish the deliveries of the drug orders.

Drug Storage Workstation:

This workstation is the center of drug circulation where manages all kinds of drug circulation operations. It reflects drug storage capacity, prices, effective periods, etc. it provides support for drug stock and discharge in the hospital. Its functions include drug addition, drug deletion, drug modification, drug query, etc.

Pharmacy Workstation:

This workstation is used for drug dispensing to the clinical department from drug storage according to the doctor orders provided by the doctor workstation. The rational use of drug prescription is checked by clinical pharmacist in this station.

Net gap:

Net gap is used for the security data exchange between hospital intranet and drug supplier internet, it uses physical isolation and security data exchange technology to realize file transfer protocol between two networks.

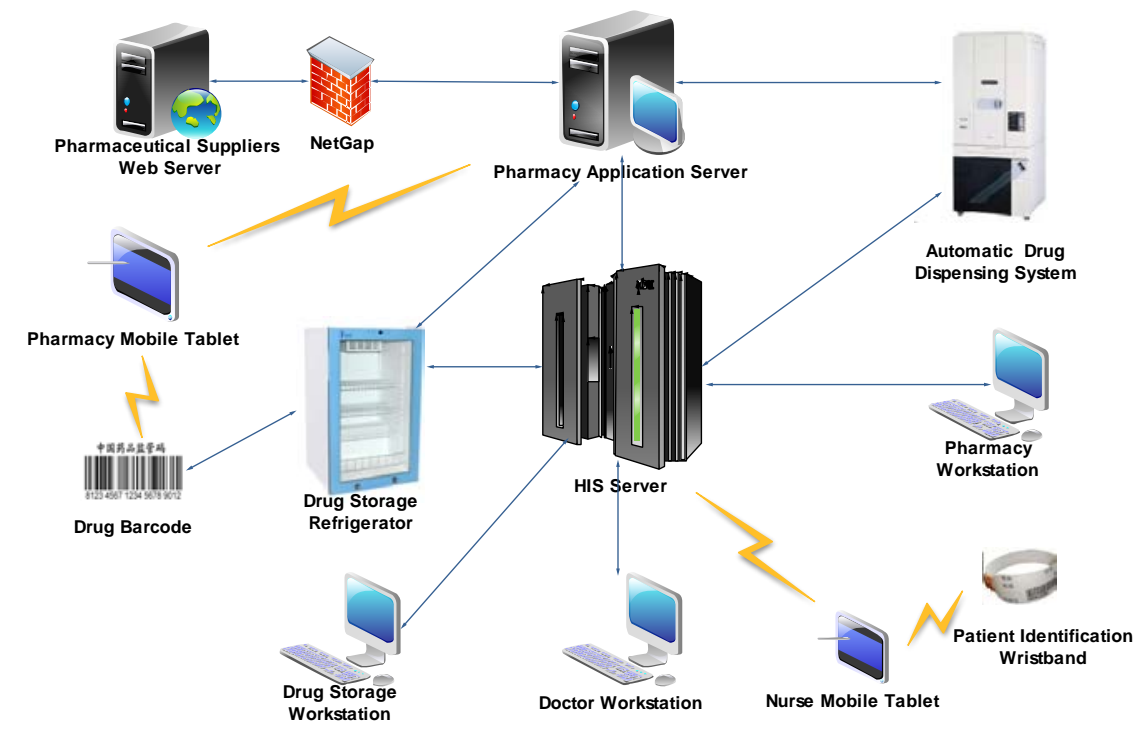

Fig. 1. System Architecture

\section{System Workflow}

The proposed system is mainly used for information process during drug purchase, request, distribution, acceptance, provision and drug inventory management in hospital ${ }^{[3] 4]}$. It can integrate the automatic equipments of hospital pharmacy and contact with the information system of pharmaceutical suppliers, It realized standard and automatic management of drug logistic and supply chain in hospital. The system integrated the information collected from medical orders, prescribing information and drug logistic in hospital information system, it can automatic generate, distribute and push drug task list, realize real-time data exchange between heterogeneous systems, 
and ensure the correctness and integrity of drug data information, provide workflow management for hospital pharmacy. The detail workflow of system is shown in figure 2 .

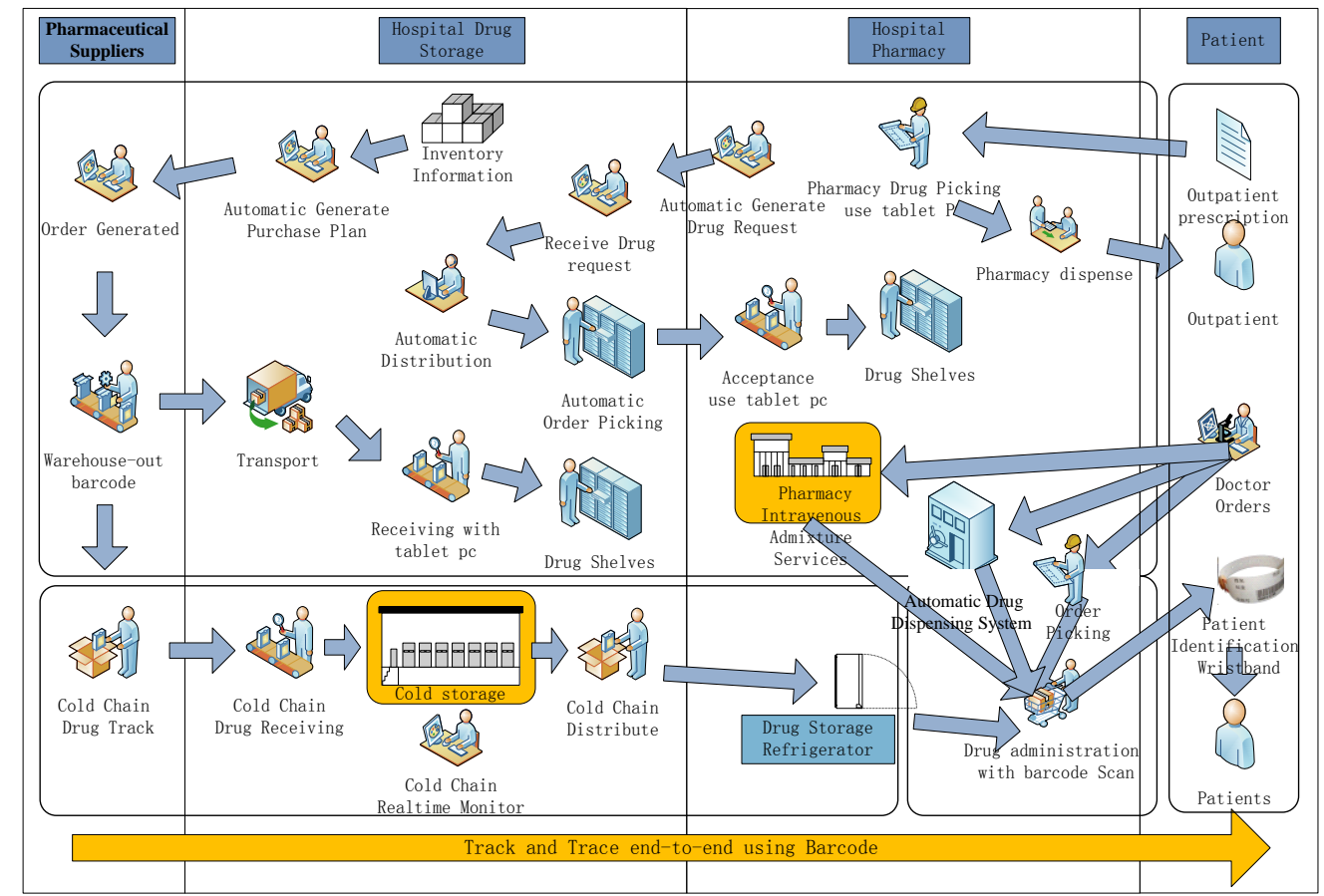

Fig. 2. System Workflow
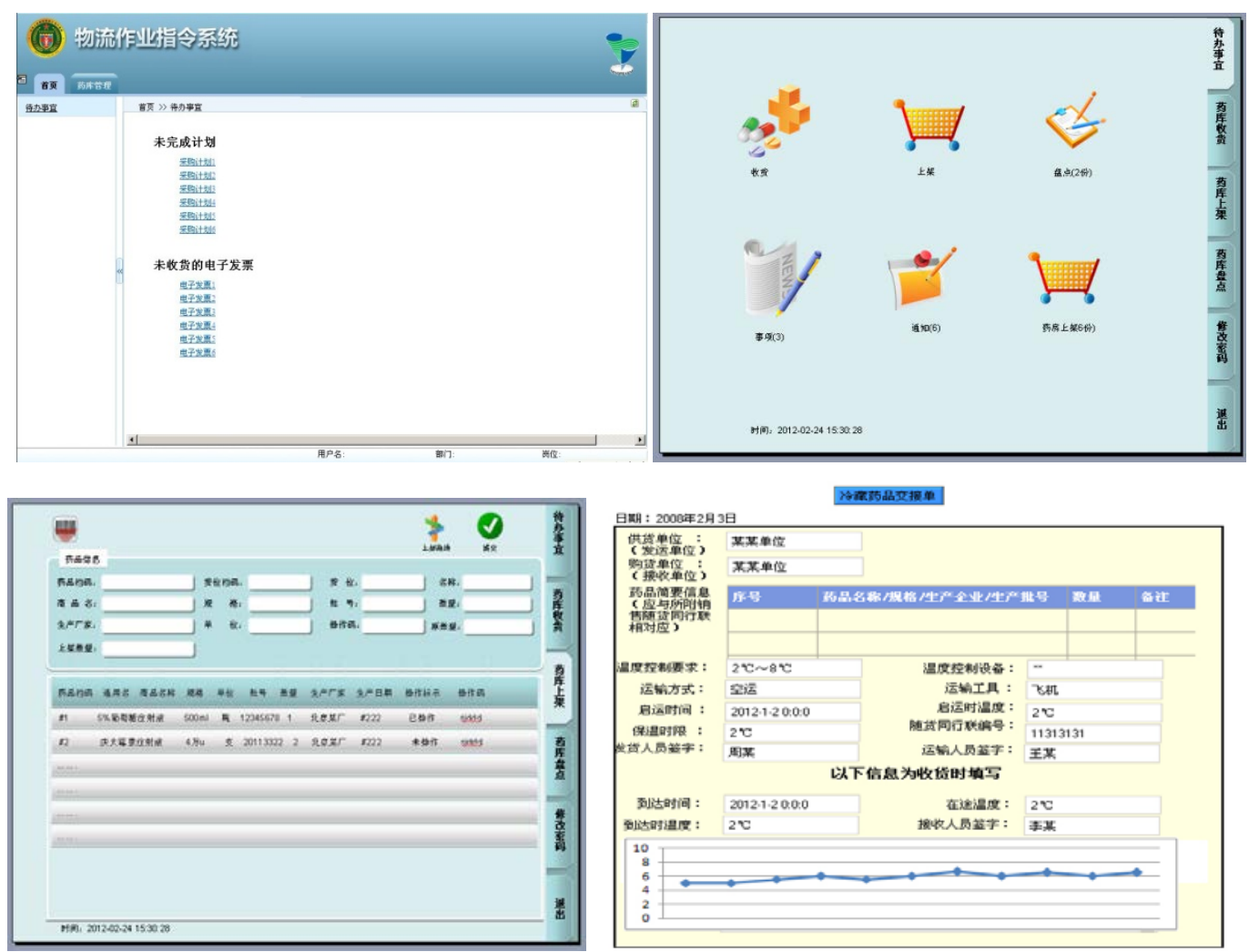

Fig. 3. System Interface

\section{Implementation of System}

We have developed the prototype implementation that was using ASP.NET, SQL server as a database management system(DBMS) and web service as data exchange interface, using barcode for identification of patients and drugs. Software architecture consists of eight major components covering each of the elements of pharmaceutical logistics and supply chain management in hospital. 
These major components are pharmaceutical supply chain management; drug inventory management; pharmacy management; inpatient area management; outpatient area management; drug cold-chain management; pharmacy intravenous admixture services system and system control management etc ${ }^{[5][6]}$.

\section{Conclusion}

In this paper, we proposed pharmaceutical logistics and supply chain management system for hospital. This system realized real-time tracing and supply chain management of all drugs in hospital by using Internet of Things and Web Services technology. This system is useful to enable cooperation amongst hospital personnel and drug suppliers by sharing drug information that is relevant to the drug prescription and supply chain which is typically distributed across multiple information systems. This system has a significant impact on information management of hospital pharmacy. Currently, security mechanisms have been implemented in the prototype system. Research is underway to enforce security at an infrastructure level.

\section{References}

[1] Yan B, Huang G. Supply chain information transmission based on RFID and internet of things[C]//Computing, Communication, Control, and Management, 2009. CCCM 2009. ISECS International Colloquium on. IEEE, 2009, 4: 166-169.

[2] Costantino N, Dotoli M, Falagario M, et al. Design and optimization of a hospital drug distribution system[J]. Proc. IPSERA, 2009.

[3] Dotoli M, Fanti M P, Meloni C, et al. Design and optimization of integrated e-supply chain for agile and environmentally conscious manufacturing[J]. Systems, Man and Cybernetics, Part A: Systems and Huans, IEEE Transactions on, 2006, 36(1): 62-75.6

[4] Singh M, Rice J B, Riquier D. Transforming the global health care supply chain[J]. MIT Center for Transportation and Logistics, 2006.

[5] Vissers J M H, Bertrand J W M, De Vries G. A framework for production control in health care organizations[J]. Production Planning \& Control, 2001, 12(6): 591-604.

[6] van Merode G G, Groothuis S, Hasman A. Enterprise resource planning for hospitals[J]. International Journal of Medical Informatics, 2004, 73(6): 493-501. 